\title{
A REDUCIBILITY CONDITION FOR RECURSIVENESS ${ }^{1}$
}

PAUL H. MORRIS

\begin{abstract}
A result due to Jockusch, equating recursiveness of a set to a reducibility condition on its jump, is sharpened.
\end{abstract}

Introduction. Unexplained notation is taken from Rogers [5]. In an unpublished proof in 1970, Carl Jockusch showed that if $A^{\prime} \leqslant_{\mathrm{btt}} \varnothing^{\prime}$ then $A$ is recursive (the converse is immediate). A short proof of an indirect nature was later obtained by Gordon Phillips, a student of Jockusch. This paper gives a fairly direct proof of a more basic result from which that of Jockusch follows immediately.

We write $A \oplus B$ for the set $\{2 x: x \in A\} \cup\{2 x+1: x \in B\}$. Following Soare [6] set $H_{A}=\left\{e: W_{e} \cap A \neq \varnothing\right\}$. In the context of $A$ co-r.e. Soare has called $H_{A}$ the "weak jump" of $A$. For general $A$ it seems appropriate to give this name to $H_{A} \oplus H_{\bar{A}}$ (if $A$ is co-r.e. and nonempty then $H_{A} \equiv H_{A} \oplus H_{\bar{A}}$ ). The relationship of the weak jump and $S$-reducibility [2] is analogous to that of the jump and Turing reducibility. $H_{A}$ has been studied by Hay [3], [4] and Soare [6], [7] and has been involved in a number of interesting relationships.

Let $t$ be the tt-condition $\left\langle\left\langle x_{1}, \ldots, x_{n}\right\rangle, \alpha\right\rangle$ (see [5, p. 110]). We denote the associated set $\left\{x_{1}, \ldots, x_{n}\right\}$ by $F_{t}$. If $\alpha(0, \ldots, 0)=0$ we say $t$ is zero-preserving.

Results.

Theorem 1. If $A$ is r.e. and $H_{\bar{A}} \leqslant_{\mathrm{btt}} \varnothing^{\prime}$ then $A$ is recursive.

Proof. Let $n$ be the least integer such that $H_{\bar{A}} \leqslant_{\text {btt }} \varnothing^{\prime}$ with norm bounded by $n$. Let $h$ be a recursive function such that $e \in H_{\bar{A}} \Leftrightarrow$ the tt-condition $h(e)$ is satisfied by $\varnothing^{\prime}$, and each $h(e)$ has norm bounded by $n$. Assume $A$ nonrecursive. Define

$$
W_{f(e, x)}= \begin{cases}W_{e} & \text { if } x \in \varnothing^{\prime} \\ \varnothing & \text { otherwise }\end{cases}
$$

Note that if $x \in \varnothing^{\prime}$ then $f(e, x) \in H_{\bar{A}} \Leftrightarrow e \in H_{\bar{A}}$. Define $W_{g(e, y)}=W_{e}$ $\cup\{y\}$. If $y \in A$ then $g(e, y) \in H_{\bar{A}} \Leftrightarrow e \in H_{\bar{A}}$. Fix $e$. Set

Received by the editors August 25, 1975.

AMS (MOS) subject classifications (1970). Primary 02F25, 02F30.

1 This work was supported by NRC grant 126-6324-32. 


$$
\begin{aligned}
& B_{1}=\left\{f(e, x): x \in \varnothing^{\prime} \text { and } F_{h(f(e, x))} \cap \varnothing^{\prime} \neq \varnothing\right\}, \\
& B_{2}=\left\{g(e, y): y \in A \text { and } F_{h(g(e, y))} \cap \varnothing^{\prime} \neq \varnothing\right\} .
\end{aligned}
$$

Note that $B_{1}$ and $B_{2}$ are r.e. Put $B=B_{1} \cup B_{2}$. We will show that $B$ is nonempty.

Observe that for any $u$, if $F_{h(u)} \cap \varnothing^{\prime}=\varnothing$, then $u \in H_{\bar{A}} \Leftrightarrow h(u)$ is not zeropreserving. We distinguish two cases:

Case 1. $e \in H_{\bar{A}}$. Then $x \in \varnothing^{\prime} \Leftrightarrow f(e, x) \in H_{\bar{A}}$. Thus if $F_{h(f(e, x))} \cap \varnothing^{\prime}$ $=\varnothing$ we have $x \in \bar{\varnothing}^{\prime} \Leftrightarrow h(f(e, x))$ is zero-preserving.

Suppose $B_{1}=\varnothing$. Then $F_{h(f(e, x))} \cap \varnothing^{\prime} \neq \varnothing \Rightarrow x \in \bar{\varnothing}^{\prime}$. Putting these together we get $x \in \bar{\varnothing}^{\prime} \Leftrightarrow F_{h(f(e, x))} \cap \varnothing^{\prime} \neq \varnothing$ or $h(f(e, x))$ is zero-preserving. This implies $\bar{\varnothing}^{\prime}$ is r.e., a falsehood. Thus $B_{1} \neq \varnothing$.

Case 2. $e \notin H_{\bar{A}}$. In this case $y \in A \Leftrightarrow g(e, y) \notin H_{\bar{A}}$. Consequently if $F_{h(g(e, y))} \cap \varnothing^{\prime}=\varnothing$ we have $y \in \bar{A} \Leftrightarrow h(g(e, y))$ is not zero-preserving. Assuming $B_{2}=\varnothing$ now gives $y \in \bar{A} \Leftrightarrow F_{h(g(e, y))} \cap \varnothing^{\prime} \neq \varnothing$ or $h(g(e, y))$ is not zero-preserving. It follows that $A$ is recursive, contrary to supposition. Here we conclude $B_{2} \neq \varnothing$.

Now let $z$ be the first element in an enumeration of $B$. Since $F_{h(z)} \cap \varnothing^{\prime} \neq \varnothing$ we can form a tt-condition $t$ with norm bounded by $n-1$ such that $t$ is satisfied by $\varnothing^{\prime} \Leftrightarrow h(z)$ is satisfied by $\varnothing^{\prime} \Leftrightarrow e \in H_{\bar{A}}$.

Redefining $h(e)=t$ we see that $H_{\bar{A}} \leqslant_{\mathrm{btt}} \varnothing^{\prime}$ with norm bounded by $n-1$, contradicting the minimality of $n$. We conclude that $A$ is recursive. Q.E.D.

Note that the above proof does not supply a decision procedure for $A$. Theorem 1 confirms a conjecture of Hay [3].

THEOREM 2. If $H_{A} \oplus H_{\bar{A}} \leqslant_{\mathrm{btt}} \varnothing^{\prime}$, then $A$ is recursive.

Proof. If $H_{A} \oplus H_{\bar{A}} \leqslant_{\mathrm{btt}} \varnothing^{\prime}$ then $A \leqslant_{\mathrm{btt}} \varnothing^{\prime}$.

By [5, Theorem 14-IX] $A$ is a Boolean combination of r.e. sets. It follows from Ershov [1] that there are r.e. sets $R_{1}, \ldots, R_{n}$ such that $R_{1} \subseteq \cdots \subseteq R_{n}$ and

$$
A= \begin{cases}\cup_{i=1}^{n / 2}\left(R_{2 i}-R_{2 i-1}\right) & \text { if } n \text { is even, } \\ R_{1} \cup \cup_{i=1}^{(n-1) / 2}\left(R_{2 i+1}-R_{2 i}\right) & \text { if } n \text { is odd. }\end{cases}
$$

We will prove the theorem by induction on $n$. For $n=1$ the result follows from Theorem 1. Suppose $n>1$. Let $f$ enumerate $R_{n}$. Let $B=f^{-1}(\bar{A})$. Then $B \leqslant_{m} \bar{A}$. Hence $H_{B} \leqslant_{1} H_{\bar{A}}$ and $H_{\bar{B}} \leqslant_{1} H_{A}$ and so $H_{B} \oplus H_{\bar{B}} \leqslant_{\mathrm{btt}} \varnothing^{\prime}$.

Let $S_{i}=f^{-1}\left(R_{i}\right), 1 \leqslant i \leqslant n-1$. Then

$$
B= \begin{cases}\cup_{i=1}^{(n-1) / 2}\left(S_{2 i}-S_{2 i-1}\right) & \text { if } n-1 \text { is even, } \\ S_{1} \cup \cup_{i=1}^{(n-2) / 2}\left(S_{2 i+1}-S_{2 i}\right) & \text { if } n-1 \text { is odd. }\end{cases}
$$

The inductive hypothesis now yields $B$ is recursive. It follows that $A$ is r.e. By Theorem $1, A$ is recursive. Q.E.D. 
Corollary (Jockusch). If $A^{\prime} \leqslant_{\mathrm{btt}} \varnothing^{\prime}$ then $A$ is recursive.

Proof. Clearly $H_{A} \leqslant_{1} A^{\prime}$ and $H_{\bar{A}} \leqslant_{1} A^{\prime}$. The result follows.

Closing remarks. In view of Theorem 2, it might be supposed that $H_{A}$ $\leqslant_{\text {btt }} \varnothing^{\prime} \Leftrightarrow A$ r.e. This is false, as is demonstrated by an elaborate construction in [3].

The author is grateful to Louise Hay and the referee for comments which have improved the presentation of these results.

\section{REFERENCES}

1. Ju. L. Ersov, On a hierarchy of sets. I, Algebra i Logika 7 (1968), no. 1, 47-74 = Algebra and Logic 7 (1968), 25-43. MR 42 \# 5794.

2. J.T. Gill and P.H. Morris, On subcreative sets and S-reducibility, J. Symbolic Logic 39 (1974), 669-677.

3. L. Hay, A noninitial segment of index sets, J. Symbolic Logic 39 (1974), 209-224. MR 50 \#6813.

4. The halting problem relativized to complements, Proc. Amer. Math. Soc. 41 (1973), 583-587. MR 48 \# 5837.

5. H. Rogers, Jr., Theory of recursive functions and effective computability, McGraw-Hill, New York, 1967. MR 37 \#61.

6. R.I. Soare, Degrees and structure of speedable sets, Abstract, January, 1974 (preprint).

7. - Automorphisms of the lattice of recursively enumerable sets, Bull. Amer. Math. Soc. 80 (1974), 53-58.

Department of Computer Science, University of Waterloo, Waterloo, Ontario, CanaDA N2L 3G1

Current address: 2901 Hillegass \#2, Berkeley, California 94705 